\title{
Scenario Design for Training Systems in Crisis Management: Training Resilience Capabilities
}

\author{
Amy Rankin ${ }^{1}$, Joris Field ${ }^{2}$, William Wong ${ }^{3}$, Henrik Eriksson $^{4}$, Jonas Lundberg $^{5}$ Chris \\ Rooney $^{6}$ \\ ${ }^{1,4,5}$ Department of Computer and Information Science, Linköping University, Sweden \\ amy.rankin@liu.se \\ henrik.eriksson@liu.se \\ jonas.lundberg@liu.se \\ ${ }^{2}$ National Aerospace Laboratory (NLR), Amsterdam, Netherlands \\ joris.field@nlr.nl \\ ${ }^{3,6}$ Department of Engineering and Information Science, \\ Middlesex University, London, UK \\ w.wong@mdx.ac.uk \\ c.rooney@mdx.ac.uk
}

\begin{abstract}
Virtual reality (VR) based training systems can be used to increase resilience in Crisis Management (CM) teams. Today's training systems largely focus on technical and procedural skills, enforcing limitations on the freedom of interaction the trainee has compared with the real world. Although these types of skills are crucial to successful response operations, the dynamic and unpredictable nature of a crisis also requires skills that are flexible, adaptive and creative. We present a variable uncertainty framework (VUF) for designing and configuring training scenarios which can be used to train capabilities such as flexibility and improvisation, aimed at increasing teams and organizations resilience. When designing a scenario using the VUF events are injected to stimulate specific training needs. By using a sense-making and command-andcontrol loop we will illustrate how specific training objectives can be targeted to train resilience capabilities.
\end{abstract}

\section{INTRODUCTION}

Crisis management $(\mathrm{CM})$ operations take place in multi-organization teams that respond to a single, or series of, event(s) that are initially outside their control. It is possible for these teams to train for their response in general terms, but in practice every event will have unique aspects that make full preparation difficult to achieve. Therefore, key aspects of crisis management training include teamwork, dealing with the situation in a 
flexible manner, and increasing skills of adaptive thinking (Bergström et al., 2011; Raybourn, 2007). These skills involve responding to what is actually taking place and adapting to the situation as required.

Resilience is a common perspective used to understand abilities associated with unexpected events in crisis management (for a review on views of resilience in disaster management, see Manyena, (2006)). Resilience is the ability to deal with changes that go outside of the designed-for system tolerances. During response operations organizations mainly strive toward maintaining established and known aspects such as policies, procedures, practices and tools. Preparation and planning are thus important activities for creating resilient organizations. However, environmental conditions such as uncertainty, complexity and dynamism make it impossible to rely solely on pre-defined structures and existing plans, as they may not match up with the on-going situation. Generic competencies, rather than well-trained procedures, are central for responding to unexpected and escalating events (Bergström et al., 2011). If some aspect fails or is inadequate to deal with the current situation, resilience competencies are central for an effective response effort.

Training CM organizations in live exercises involving multiple agencies are costly in terms of time and funding, making digital technology an important new source for training. Training systems, on the other hand, often have limitations to train adaptive behaviour as they are context-specific and anchored to the technical environment (Dekker et al., 2008). Using training systems for training flexibility in unpredictable and unforeseen situations therefore poses challenges both to technology and to our imagination.

We present the variable uncertainty framework (VUF) as a means to configure adaptive and flexible scenarios in training systems. The VUF is currently being used in an EU project (CRISIS), to design and configure scenarios in a VR environment for crisis management training. To target specific training objectives using the VUF events are injected into scenario in various. To create scenarios targeting specific training objectives it is necessary to identify how different types of events affect the ongoing response operation. We present a sense-making and control loop as a useful tool to illustrate how resilience capabilities affect the CM system.

\section{VARIABLE UNCERTAINTY FRAMEWORK}

Training scenarios have the problem of being time consuming to create, and are often played out in a linear and rigid manner. The VUF allows for a variety of ways to create, adapt and change a scenario to create uncertainty and complexity. This task is done by bringing together three important dimensions of real-world emergencies that can be manipulated and controlled in the virtual training environments: (1) situational complexity, (2) the number of events occurring, and (3) the randomness of the presentation of these events (Figure 1) (Wong, Rankin, \& Rooney, forthcoming). These dimensions enable decomposition in scenario building blocks, which can be combined and configured to address particular training objectives. 


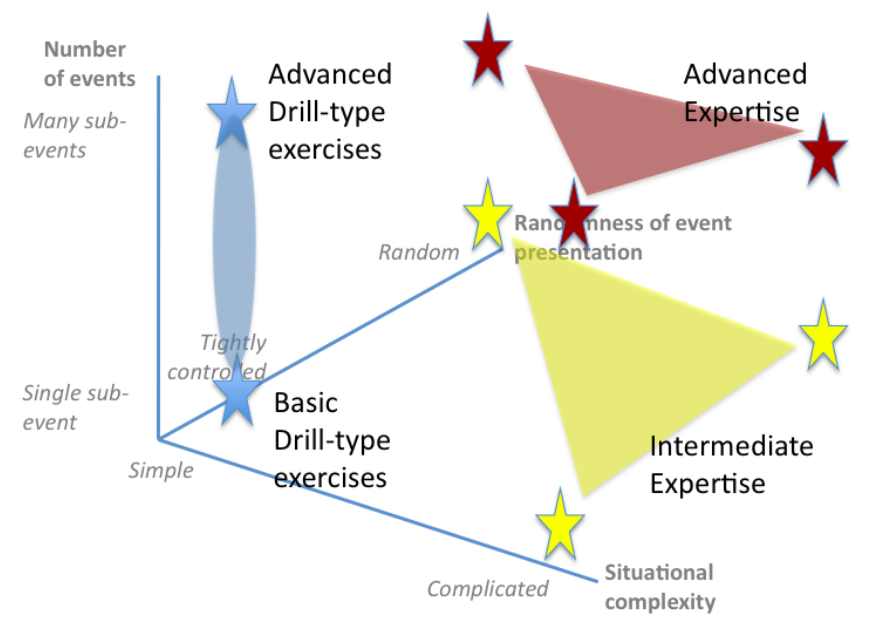

Figure 1. Variable uncertainty, achieved by combining basic scenario building blocks in different ways (Wong, Rankin, Rooney, forthcoming).

For the purpose of configurability, situational complexity is seen as the extent of the relationships between events and the objects of the situation, and the nature of the dependencies between them. Interrelated events cause higher complexity as the uncertainty and unpredictability of the situation increases. The number of events refers to the amount of events occurring simultaneously in the simulation. The randomness of events refers to the predictability with which events and objects occur.

By controlling these three dimensions, an instructor can design scenarios that are either basic drill oriented, with loosely coupled events, or advanced scenarios where events are combined in un-predictable ways to increase time-pressure and complexity. Playing out events in a predictable fashion is used for training basic-drill exercises or when it is desired to control and train for specific behaviors among group trainees as they respond to a situation. Designing scenarios for training complex task is, however, more of a challenge as many variables must be taken into account, and the dependencies between the events become too complex to oversee. To illustrate how certain aspects of the organization are affected, depending on the type of injected event, a sense-making and control loop is presented (Figure 2). The loop describes how events apart from the main crisis scenario can be added to target specific training objectives, in particular those focused on resilience capabilities. To avoid confusion with all events in the scenario, we will name these additional events injects.

\section{SCENARIO DESIGN USING A SENSE-MAKING LOOP}

Figure 2 illustrates some central aspects of sense-making and control in variable uncertainty scenarios. 


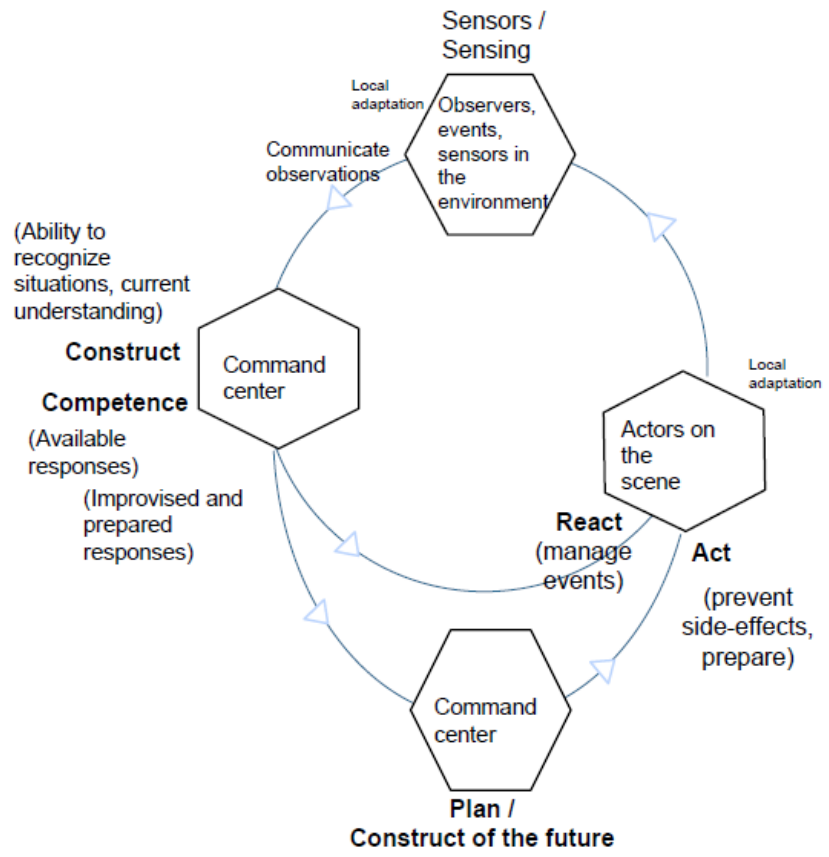

Figure 2. Central aspects of sense-making and control in variable uncertainty scenarios

The model is based on previous models of team cognition and command-and-control (e.g. DOODA (Brehmer, 2007), COCOM (Hollnagel \& Woods, 2005)). The basic cyclical model is commonly used to illustrate the relationship between the feedforward and feedback loops of command-and-control in socio-technical systems. The response teams must have some means of sensing events, either directly or by some team member, or with the help of some other sensor (Sensor/Sensing, Figure 2). This information is conveyed to commanders at different levels in the organization. The team will rely on collective experience and understanding to interpret the situation (Construct/Competence, Figure 2). A central part of crisis management is planning for future events and actions, taking into account eventualities, side-effects and possible outcomes of a situation (Plan/Construct for the future, Figure 2). Based on the interpretation, actions are taken to manage the operation, either as a direct reaction to an ongoing situation or as part of a plan for the future (Act/React). The action will alter the system state, and new information is provided via the sensors. An event will often have an effect on the entire command-and-control-loop. For example, if additional casualties are discovered by a fire fighter (Sensor/Sensing), this information is reported back to the command post (construct/competence), where staff members have to allocate resources to deal with the additional casualty (Act, React). This re-allocation of resources may cause other plans to be revised.

By inserting injects into different parts of the loop, different capabilities can be targeted to challenge different aspects of the organization and team's resilience (see Figure 3 and Table 1). 


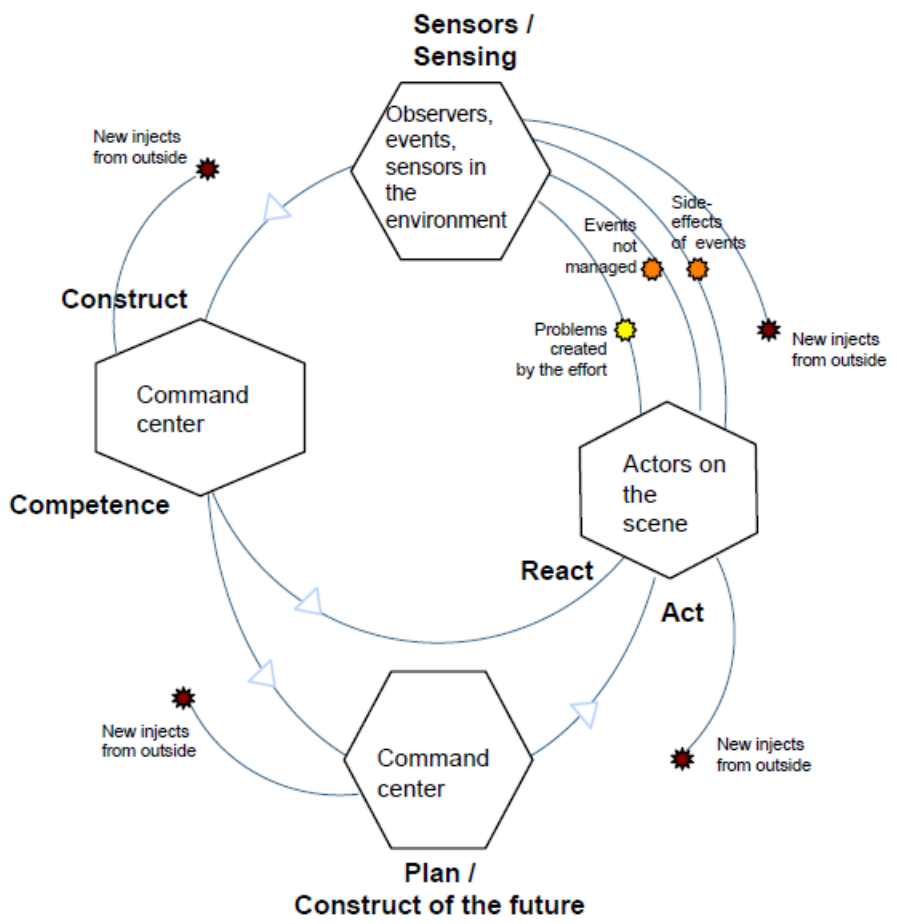

Figure 3. Designing variable uncertainty scenarios using injects

For example, to train field personnel in detecting unanticipated events, injects will be inserted in the sensor/sensing, such as a new fire igniting or a casualty's health rapidly deteriorating. The injects can be more or less visible and easy to detect. At the construct level the command staff's task is to interpret the ongoing situation and make decisions on what actions to take based on the incoming stream of information. Injects to this part of the system sense-making can be done either via the sensors (though this cannot be controlled if the sensor is another trainee) or directly into the construct level, for example, a phone call from local media asking questions and providing new information. Adding multiple injects will increase the workload (i.e. number of events, Figure 1). Further, if both injects are implemented at the same time and the information from the local media is contradictory to what is being reported by the team sensors, this will increase the level of uncertainty (i.e. randomness of events, Figure 1). Plan/construct of events (Figure 3) illustrates the commander's plans for future events, to foresee risks and possible side effects. Altering this phase of the sense-making loop can be done by, for instance increasing/decreasing resources. For example, a Medevac helicopter which should be on its way reports that it will be 45 minutes late, causing the command staff to re-plan the care and transportation of some casualties. Inserting an inject into the act/react phase of the sense-making loop (e.g. vehicle failure, radio communication failed) will alter the state of the system, requiring problem diagnosis by sensors and a new construct for the command team level. 
Table 1. Summary of capabilities and injects in the sense-making loop

\begin{tabular}{|l|l|l|}
\hline Part of sense-making loop & Capabilities & Sample Injects \\
\hline $\begin{array}{l}\text { Sensors/sensing } \\
\text { Observations or other sensors } \\
\text { in the environment }\end{array}$ & $\begin{array}{l}\text { Problem detection, vigilance, } \\
\text { local adaptations, monitoring }\end{array}$ & $\begin{array}{l}\text { Fire igniting, casualties health } \\
\text { deteriorating, }\end{array}$ \\
\hline $\begin{array}{l}\text { Construct/Competence } \\
\text { Interpretation of current } \\
\text { system state }\end{array}$ & $\begin{array}{l}\text { Information management, sort } \\
\text { and prioritize, goal } \\
\text { formulation }\end{array}$ & $\begin{array}{l}\text { Conflicting information, large } \\
\text { amounts of information }\end{array}$ \\
\hline $\begin{array}{l}\text { Plan/ Construct of the } \\
\text { future }\end{array}$ & $\begin{array}{l}\text { Plan for future events, } \\
\text { (possible outcomes and side- } \\
\text { effects) flexibility, adaptation }\end{array}$ & $\begin{array}{l}\text { Alter system status, e.g. } \\
\text { increase or decrease } \\
\text { resources/time }\end{array}$ \\
\hline $\begin{array}{l}\text { React/Act } \\
\text { React } \text { on current } \\
\text { understanding of system state, } \\
\text { Act } \text { on the overall plan }\end{array}$ & $\begin{array}{l}\text { Manage operation in the real } \\
\text { world, implement decisions } \\
\text { made }\end{array}$ & $\begin{array}{l}\text { Vehicle failure, phone radio } \\
\text { communication failure, water } \\
\text { hose not long enough }\end{array}$ \\
\hline
\end{tabular}

In addition, new events may be created as a result of how the team manages the ongoing situation. The loop in Figure 3 illustrates the efforts to manage the ongoing events, some of which may not be solved, meaning that when new events occur, the previous events remain to be fixed as well (between Act/React and Sensor/Sensing). The outer circle (Plan/Construct of the future) consists of attempts to foresee effects of the current situation, which means that the team can intervene and stop them from occurring (e.g. if electrical power is restored to system $\mathrm{X}$, there will be no flooding at $\mathrm{Y}$ ). The number of side-effects, the difficulty in discovering cues to suggest that they might occur, and the need for a particular expertise to understand the implications of cues, is important aspects of situational complexity (Figure 1).

To target generic capabilities for increasing resilience injects affecting Plan/Construct of future should be used, i.e., to foresee possible events and side effects. If actions played out "on the scene" do not have the desired outcome, the preparedness of commanders is central. Using injects in the scenario to alter the current plan of operation therefore challenges the organization to adapt to the current situation. Injects can be positive, e.g., more resources become available or more time is provided. Taking (identifying) opportunities as they arise is an important part of resilience performance. The injects can also be negative, e.g. lessened resources, system failures or increased time pressure. In this case the team will have to be flexible. They must improvise, re-plan and prioritize to manage the situation, and at the same time minimize unwanted side effect. If not managed correctly, the crisis will keep escalating. Note that injects can be injected to any part the sense-making model and still target another part.

To summarize, designing complex and flexible scenarios in CM training systems requires an understanding of the relationships between the available selection of events in the system and the training objectives for the trainee. One way to illustrate how different events (injects) affect the scenario is to link them to a command-and-control 
sense-making loop. This can aid the scenario planner to target certain capabilities such as problem detection, situation understanding, flexibility, improvisation and planning. Implementing injects focused on the same event will increase the coupling of events, which creates more complex scenarios using, for instance, time-pressure, ambiguity and resource prioritization.

\section{CONCLUSIONS}

Improving resilience in complex situations requires a multifaceted training approach. Designing scenarios to match training needs in CM teams requires an understanding of how events and complexity are related to the task and management of the CM team. By linking theories of sense-making and control to the variable uncertainty framework (VUF) for designing scenarios we have created a basis from which complex and adaptive scenarios can be designed. The framework illustrates how training systems can have a great potential to improve skills critical for managing the dynamic and unpredictable nature of a crisis and, hence, increasing the resilient performance of teams.

\section{ACKNOWLEDGEMENTS}

This research has been funded by the European Commission's FP7 Framework Programme for Security Research, Grant Agreement Number FP7-24247.

\section{REFERENCES}

Bergström, J., Dahlström, N., Dekker, Sidney, \& Petersen, K. (2011). Training Organizational Resilience in Escalating Situations. In E. Hollnagel, J. Pariès, D. Woods, \& J. Wreathall (Eds.), Resilience Engineering in Practice. Ashgate Publishing Limited.

Brehmer, B. (2007). Understanding the functions of $\mathrm{C} 2$ is the key to progress. The International C2 Journal, 1, 211-232.

Dekker, Sydney, Dahlström, N., Van Winsen, R., \& Nyce, J. (2008). Crew Resilience and Simulator Training in Aviation. In E. Hollnagel, C. P. Nemeth, \& Sidney Dekker (Eds.), Resilience Engineering Pespectives: Vol 1 Remaining Senseitiv to the Poosibility of failure. Aldershot: Ashgate.

Hollnagel, E., \& Woods, D. D. (2005). Joint Cognitive Sysytems: Foundations of Cognitive Systems Engineering. Group. Boca Ranton: CRC Press, Taylor \& Francis Group.

Manyena, S. B. (2006). The concept of resilience revisited. Disasters, 30(4), 433-50.

Raybourn, E. (2007). Applying simulation experience design methods to creating serious game-based adaptive training systems. Interacting with Computers, 19(2), 206-214.

Wong, W., Rankin, A., \& Rooney, C. (forthcoming). The variable Uncertainty Framework. Hendon: Middlesex University. 